\title{
Rhythms in Marine Invertebrates: Managing Intertidal Life alongside Daily Program
}

\author{
Neelu Jain Gupta* \\ Department of Zoology, MMH College, India \\ *Corresponding author: Neelu Jain Gupta, Department of Zoology, MMH College, Ghaziabad, India
}

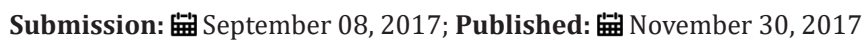

\begin{abstract}
The cyclicity of moon acts as a synchronizing cue to the sea life through tides. It exerts selective pressure such as water pressure, air exposure etc. causing wide affects ranging from timing of reproduction to migration. Although it is established that intertidal cyclicityis pervasive, the extents of its interplay with daily changes in light and dark cycles is not clearly understood. Chronobiological studies on marine invertebrate life are important to unveil the modulation of the interaction of daily and tidal rhythms with the course of evolution. Further, melatonin, 'the hormone of darkness' is a phylogenetically conserved molecule known for sleep-regulation in vertebrates. The characterization of melatonin in invertebrates has reiterated recognition of putative role of melatonin as an antioxidative protectant involved in the detoxification of destructive radicals, besides a transducer of photoperiodic information. In recent decade, molecular investigations of invertebrate clocks have suggested complex biological clocks-controlled by multiple clock output pathways with plasticity daily downstream events.
\end{abstract}

Keywords: Intertidal; Invertebrate; Evolution; Melatonin; Rhythms

\section{Introduction}

Earth's rotation on its axis generates predictable environmental ameliorations in light and temperature, helping organisms to keep a track of time on a daily basis. The alternation of light and dark phases renders survival advantage to organisms as a circadian timer and Earth's tilt on its axis, while it revolves around the Sun further allows the organisms to adjust to seasonal changes in relative span of day and night (also known as annual cycle of day length) providing a seasonal timer as well. The environmental ameliorations differ among terrestrial and marine environments. While sun and earth provide predominant environmental cues for adaptations, the geophysical affects of moon and its revolution around earth have pronounced affects on marine life.

The marine ecosystem is subjected to temporal fluctuations. Marine life constrained by additional cyclic phenomena than terrestrial life as the rhythms of moon largely affect water in form of tides thus the tidal rhythms. Moon revolves around the earth in 27.29 days, but Earth being in a rotating state, the moonrise on earth's surface has a daily delay of 50.5 minutes making a lunar day of 24.84 hours. A lunar month is 29.5 days. During a full moon, moon rises at dusk and sets at dawn; the reverse is true for new moon. Clear sky light luminance on earth from full moon to new moon ranges from $1.83 \times 10^{-1}$ to $1.8 \times 10^{-4}$ micro-watts/sq.cm. Tidal phenomena is a primary affect of lunar cycle, the tidal maxima and minima being potential time-keeping source to marine animals. A difference of duration between lunar tide cycle (24.84-hour) and solar tide cycle (24-hour) leads to alternation/ opposition of amplification of effects of the moon and sun causing spring and neap tides every 14.8 days. Other factors affecting marine environment include water pressure and air exposure at different sea depths.

Marine animals inhabiting intertidal regions live under tremendous selective pressure due to pervasive cyclic change between land and water habitats [1,2]. They need to temporally adjust their physiology and behavior to the tidal cycle, which is known as circa tidal rhythm. Less is known about molecular basis of circa tidal timekeeping. Many intertidal zone inhabiting invertebrates like fiddler crabs exhibit circa tidal rhythms in spawning or larval hatching synchronizing reproductive success to spring tides. An organism synchronizing to both high tides in one lunar month is said to have semi lunar rhythms. Till date it is not understood whether the circadian and circa lunar (circalunidian) clock could have a similar phylogenetically conserved origin close to circadian machinery or circa tidal clock is an independent clock system with different molecular mechanism [3].

A few studies suggest that molecular gears of the biological clocks mediating tidal rhythms act differently from evolutionarily conserved systems mediating circadian rhythms. Although, evolution ensured distinct clockwork mechanisms to manage intertidal life alongside the daily program, melatonin, 'the hormone of darkness,' one of the oldest among the phylogenetically conserved biological molecules captivated attention. This pleiotropic agent is 
highly diffusible yet conserved sleep-regulating hormone secreted at night by the pineal organ in vertebrates from fish to mammals. The principle significance of melatonin in non-vertebrate species remained uncertain as most investigators focused on characterization of the specific role of melatonin as transducer of photoperiodic information. This brief review discusses some key aspects of rhythmic invertebrate life in the sea with a mention of melatonin as highly conserved biomolecules with multitude of functions during the course of evolution.

\section{Rhythms in Invertebrates}

\section{Protozoa}

Spindler M et al. [4] has reported a synodic lunar cycle in reproduction of foraminifer Hastigerina pelagica. In Open Ocean all individuals underwent gametogenesis synchronized to 3-7 days after full moon. In laboratory, ability to reproduce depended on time of lunar cycle when individuals were collected from sea. A collection of cultures close to time of reproduction enabled gametogenesis, while collection made more than 13 days away from reproductive phase abolished the ability to reproduce (lunar periodicity of reproduction in the planktonic foraminifer Hastigerina pelagica [4]. Ciliates (e.g., autotrophic Mesodinium rubrum) exhibit daily movements called reverse DVM (down at dusk, up at dawn). Dinoflagellates are unicellular protists known to "bloom" during the warm months of summer.

Dinoflagellates became primary protists for recognition of putative role of melatonin as an antioxidative protectant involved in the detoxification of destructive radicals. In a dinoflagllate species, Lingulodinium polyedrum (syn. Gonyaulax polyedra), melatonin exhibits oscillation in a circadian fashion, with a nocturnal peak that is down regulated in day. While photoperiodic responses were studied in L. polyedrum, other dinoflagellate species were investigated for induction of asexual cysts. In these protozoa, cyst induction by short-days occurs under specific temperature conditions: melatonin, 5-MT (5 methoxy-tryptamine), however, strongly induces encystment, overrides photo- or thermoregulation that otherwise is permissive for circadian rhythmicity. The cystinducing effects of exogenously administered 5-MT is caused by cytoplasmic acidification involving proton translocation [5].

\section{Porifera}

Tidal rhythms in sponges are feely addressed. Tethya wilhelma, a sponges species displays rhythmic body contractions. In 2004, a differential, quantitative data on sponge behaviour was given using digital time lapse imaging and semi-automated image analysis. The sponges could reduce their body volume by up to $73.3 \%$ during contraction cycle. The contraction cycle had four phases with a characteristic pattern, permitting analysis of the kinetics of contraction and expansion. The sponge contractions displayed daynight periodicity with contraction cycles mostly restricting to dark hours. The results also suggested T. wilhelma as a model organism for studying a neural signal transduction and integration during early metazoan evolution [6]. Müller WE et al. [7] studied some elements of a postulated circadian clock circuit in siliceous demosponge Suberites domuncula suggesting cryptochrome as a photo sensor. They demonstrated thatinformation-transduction system involving generation of light (luciferase), photon transmission (through the siliceous spicules), and photon reception (cryptochrome), all occurred in the same individual. They proposed that this photoreception/photo transduction process might function as a nerve-cell-like signal transmitting system. The sponges contained the molecular circadian clock protein nocturnin, which is highly expressed in the dark. This finding on siliceous demo sponge supported that these lowest metazoans had elements of a circadian rhythm, characteristic of higher metazoans.

\section{Coelenterata}

The reef coral Pocillopora damicornis breeds with a lunar rhythm albeit throughout the year. Interestingly tides have no role to play, but in winter, breeding relates to full moon while in summer to new moon [8]. Corals also present an example of meta-organism (termed a symbiotic association) where in synchronization between coral and photosynthetic endosymbiont circadian clocks renders complex diurnal, lunar, and annual rhythmic responses. Their central metabolism is dually regulated by the hypoxia-inducible factor in coral as well as the plasticity of regulation downstream of the circadian clock [9]. At molecular level, [10] has reported bluelight-sensing photoreceptors, cryptochromes, in the reef-building coral Acropora millepora in which cryptochromes cry1 and cry2 were expressed preferentially in light. This enabled explanation for synchronization behaviors such as mass spawning as the cry2 expression increased on full moon nights versus new moon nights. These results elaborate phylogenetically broad roles of these ancient circadian clock-related molecules in the animal kingdom [10]. Another coelenterate, Actinia equina, a sea anemone exhibits tidal rhythm in its expansion and contraction, although this rhythm persisted in the laboratory for several days [11].

\section{Platyhelminthes}

Acoel flatworm Symsagitiffera roscoffensis was first marine animal studied for existence of circatidal clocks in marine organisms. These worms inhabit shallow coastal areas of English Channel, having obligate endosymbiotic association with the microalga Tetraselmis convolutae. These emerge during low tides and burrow into darkness of the sand with the rising tides. Kearn $\mathrm{G}$ et al. [12] has studied daily hatching rhythm in the monogenean skin parasite Entobdella soleae, and its alteration with host Solea solea activity [12].

\section{Annelida}

Annelids, specifically, Platynereis, the marine rag worm, is one the most studied annelid that possesses endogenous circadian and circalunar (monthly) clocks. The worm's forebrain also possesses a circalunar clock, which is entrained by nocturnal light. This monthly clock regulated maturation and persists even when circadian clock oscillations are disrupted by the inhibition of casein kinase. Both circadian and circalunar clocks converged on the regulation of 
transcript levels. Furthermore, the circalunar clock changed the period and power of circadian behaviour, although the period length of the daily transcriptional oscillations remains unaltered. Tessmar-Raibleand colleagues 2013 concluded that a second endogenous non-circadian clock can influence circadian clock function [13]. Melatonin signaling is also well studied in platyneries [14]; it plays a role in the circadian control of ciliary swimming to adjust the vertical position of zooplankton in response to ambient light. The burrow emergence activity in another wild caught rag worm Nereis virens Sars is associated with food prospecting and was tested under photoperiodic (LD), free-running LL conditions and simulated tidal cycles (STC) in laboratory. Activity cycles in $N$. virens at the population level were robust, particularly labile at the individual level, suggesting complex biological clock-control with multiple clock output pathways [15] With respect to eye evolution, Platynerieshas two types of photoreceptors, with distinct opsins (light sensing molecules) and it also coexisted in Urbilateria, the last common ancestor of insects and vertebrates. The two cell types: A rhabdomeric photoreceptor cell is present in the eyes and ciliary photoreceptor cells in the brain. Of these rhabdomeric photoreceptor cells represent insect eye while ciliary photoreceptor cells represent vertebrate eyes. Both photoreceptors coexist in Platyneries and have distinct architecture and ability to transduce the light signal by different phototransductory cascades. The ciliary photoreceptor cells can even use a photopigment closely related to vertebrate rod and cone opsins [16].

\section{Arthropoda}

Arthropoda is the most varied strata among invertebrates, which have invaded diverse habitats. Although, instances for lunar rhythms are reported in marine arthropods, there is much scope for documentation. Soren and colleagues described exogenous cycles and endogenous rhythms associated with DVM of the ecologically important and highly abundant planktonic copepod Calanus finmarchicus. Interestingly in captivity, C. finmarchicus showed circadian rhythms of DVM, metabolism, and most core circadian clock genes (clock, period1, period2, timeless, cryptochrome2, and clockwork orange). This is first reported the field investigation of clock genes in a marine species which coupled clock gene measurements with laboratory and field data on DVM. The study implied a high degree of causality between clock gene expression and daily biomass migrations of copepods. Circadian clocks increase zooplankton fitness by optimizing the temporal trade-off between feeding and predator avoidance, especially when environmental drivers are weak or absent [17].Clocks are also involved in orientation of migration in marine crustaceans [18].

Tidal rhythmicity in crabs is studied for over 50 years now. Williams BG et al. [19] studied tidal rhythms on laboratory reared crabs, confirming persistence tidal rhythmicity and that it may be inherited. They postulated that tidal variables like hydrostatic pressure, temperature changes and periodic immersion finetune activity with tides. Another early reported example of lunar rhythm is that of Clunio marinus, marine midge, tidal chironomid (Chironomidae, Diptera, Insecta). The larval insects live among intertidal algae, while the adults emerge during low waters. The adult emergence occurs with semi lunar periodicity, at the spring low tide. But populations in the Black Sea were found to emerge and copulate when offshore winds drive the water level down and expose the algae. Apparently, chironomids responded to tidal fluctuations, but the persistence of tidal rhythm in laboratory reared young chironomids in synchrony with their parental populations confirmed tidal rhythms [20]. Kaiser TS et al. [21] investigated the genetic basis of population variation in clock properties by constructing the first genetic linkage map for this species, and performing quantitative trait locus (QTL) analysis on variation in both lunar and diurnal timing. The genome had a genetic length of 167-193 centimorgans as estimated by flow cytometry. Mapping of clock genes and light receptors identified ciliary opsin2 (cops2) as a candidate to be involved in both lunar and diurnal timing; cryptochrome 1 (cry1) as a candidate gene for lunar timing; and two timeless (tim2, tim3) genes as candidate genes for diurnal timing. As a pioneer study, this QTL analysis provided a unique account of molecular analysis of the lunar clock.

Intertidal organisms may employ circatidal rhythms to track the tidal cycle, but tidal patterns may vary within a species range and necessitate adaptation to the local tides. Another popular model organism for study of circatidal rhythms in populations of the eastern Pacific is fiddler crab Uca princeps. Rhythmicity in continuously recorded locomotors activity of individual crabs held under laboratory conditions was described by cosinor and periodogram methods of time-series analysis. Both daily and circatidal rhythms were found in crabs exposed to light-dark cycles. Crabs exhibited bimodality in activity, peaking mid-morning and nocturnally with varying degree. Overall, the study suggested that populations of $U$. princeps used both daily and circatidal timing systems to track local forms of the tide generated by their wild conspecifics at natural locations [22]. Another study on shore crab Carcinus maenas in North Wales, abounding in intertidal zones. Peaks of moulting occurred in juveniles around expected times of high tides, with few crabs moulting at other times. The circatidal moulting patterns were similar in crabs collected at different stages of the neap-spring cycle. For C. maen as early juveniles, which remain in the high intertidal even when tides recede, anticipation of the rising and falling of tides through endogenous physiological programming to avoid ecdysis at the time exposed to air was shown to have clear adaptive value. The study reported coupling of circatidal and circasemilunar moulting rhythms, and their endogenous control in a crustacean [23]. Alifierakis NS et al. [24] studied special significance of tidal cycle for pelagic egg-laying periwinkles, Littorina littorea in four estuary sites. Females without the tidal experience released few eggs with little relation to the lunar month. Those in the tidal regime released more than four times the total number of eggs and released far more in periods of new and full moon than in periods of half-moon. They concluded that a twoweekly rhythm related egg release to the spring-neap tide cycle and that this rhythm required a tidal cycle of immersion and emersion for its maintenance. In another study, octopuses, 0 . vulgaris, the inhabitants of the littoral zone of marine environments were 
placed in constant conditions to document free running activity with circadian components. Octopuses used light inefficiently as a zeitgeber for activity but overall results suggested the presence of an endogenous circadian rhythm in O. vulgaris [25]. Newcomb JM et al. [26] studied daily rhythms of activity in the nudibranch Melibe leonina, to investigate which of these rhythmic behaviors a circadian clock controls and whether extraocular photoreceptors in the eyes or optic ganglia of Melibe were used to synchronize to lightdark cycles. All animals including eyeless animals expressed a daily rhythm of locomotion, with more locomotion at night and detect light using extraocular photoreceptors. The locomotion in Melibe was influenced by both ocular and extraocular photoreceptors, although eyes had greater influence on the expression of circadian rhythms.

\section{Echinoderms}

Coral-eating 'crown-of-thorns' starfish exhibit sporadic outbreaks to spatial and temporal variation in reproductive and settlement success. Environmental cues synchronize neuro hormonal endogenous rhythms regulating gamete release etc. These environmental cues act as spawning 'inducers' by causing the release of hormones (gonad stimulating substance) in sensitive males, while biological cues (pheromones) from released sperm, in turn, act as spawning synchronizers by triggering a hormonal cascade resulting in gamete shedding by conspecifics. Given the immediate temporal linkage between the timing of spawning and fertilization events, variability in the extent and synchronicity of gamete release will significantly influence reproductive success and accounts for fluctuations in the abundance of crown-of-thorns starfish [27]. In a study involving the presence of melatonin and enzymes of its pathway in the sea star Echinaster brasiliensis, it was demonstrated that E. brasiliensis produced endogenous melatonin in the gonads, under a circadian pattern with a nocturnal peak of production. The precursor enzymes arylalkylamine $\mathrm{N}$-acetyltransferase (AANAT) and tryptophan hydroxylase (TPH) regulated the melatonin production [28].

Sea urchin is model organisms to investigate behavioral evidence for photosensitivity, as their light sensing has remained an enigma. Genome information and ultra structure characterization of the purple sea urchin (Strongylocentrotus purpuratus) revealed photoreceptor cells PRCs to be of a microvillar receptor type. These echinoderms, in contrast to chordates, deployed a microvillar, r-opsin-expressing PRC type for vision, a feature limited to protostome animals. Sea urchin PRCs also lacked any associated screening pigment. The PRC axons connected to the animal internal nervous system, suggesting an integrative function beyond local short circuits [29]. Variation of activity behaviour patterns of sea cucumber; Apostichopus japonicas. Dong G et al. [30] were investigated using light intensity protocols. The daily behavior of A. japonicus was influenced under strong light conditions affecting feeding, but food proportions were not decreased with the increase of light intensity [30]. The above account substantiates the fact that based on adaptive needs; the marine invertebrates evolved a unique interplay of tidal and daily rhythms essential to their survival.

\section{References}

1. Finger man M (1957) Lunar rhythmicity in marine organisms. The American Naturalist 91(858): 167-178.

2. McDowall RM (1969) Lunar Rhythms in Aquatic Animals: A General Review. Tuatara 17(3): 133-143.

3. Iglesia HDL, Johnson CH (2013) Biological Clocks: Riding the Tides. Curr Biol 23(20): R921-R923.

4. Spindler M, Hemleben C, Bayer U, Be AWH, Anderson OR (1979) Lunar periodicity of reproduction in the planktonic foraminifer Hastigerina pelagica. Mar Ecol Prog Ser 1: 61-64.

5. Hardeland R, Balzer I, Poeggeler B, Fuhrberg B, Uría H, et al. (1995) On the primary functions of melatonin in evolution: mediation of photoperiodic signals in a unicell, photooxidation and scavenging of free radicals. J Pineal Res 18(2): 104-111.

6. Nickel M (2004) Kinetics and rhythm of body contractions in the sponge Tethya wilhelma (Porifera: Demospongiae). J Exp Biol 207(Pt 26): 45154524

7. Müller WE, Schröder HC, Pisignano D, Markl JS, Wang X, et al. (2013) Metazoan circadian rhythm: toward an understanding of a light-based zeitgeber in sponges. Integr Comp Biol 53(1): 103-117.

8. Moore DR (1958) Notes on Blanquilla, the most northerly coral formation in the western Gulf of Mexico. Publ Inst Mar Sci 5: 151-155.

9. Sorek M, Díaz-Almeyda EM, Medina M, Levy O (2014) Circadian clocks in symbiotic corals: the duet between Symbiodinium algae and their coral host. Mar Genomics 14: 47-57.

10. Levy O, Appelbaum L, Leggat W, Gothlif Y, Hayward DC, et al. (2007) Light-responsive cryptochromes from a simple multicellular animal, the coral Acropora millepora. Science 318 (5849): 467-470.

11. Cloudsley-Thompson JL (1961) Rhythmic Activity In Animal Physiology And Behaviour. Academic Press, London and New York, USA, p. 236.

12. Kearn G (1973) An endogenous circadian hatching rhythm in the monogenean skin parasite Entobdellasoleae, and its relationship to the activity rhythm of the host (Soleasolea). Parasitology 66(1): 101-122.

13. Zantke J, Ishikawa FT, Arboleda E, Lohs C, Schipany K, et al. (2013) Circadian and circalunar clock interactions in a marine annelid. Cell Rep 5(1): 99-113.

14. Tosches MA, Bucher D, Vopalensky P, Arendt D (2014) Melatonin signaling controls circadian swimming behavior in marine zooplankton. Cell 159(1): 46-57.

15. Last KS, Bailhache T, Kramer C, Kyriacou CP, Rosato E, et al. (2009) Tidal, daily, and lunar-day activity cycles in the marine polychaete Nereis virens. Chronobiol Int 26(2): 167-83.

16. Arendt D, Tessmar RK, Snyman H, Dorresteijn AW, Wittbrodt J, et al. (2004) Cilliary photoreceptors with a vertebrate-type opsin in an invertebrate brain. Science 306(5697): 869-71.

17. Sören NH, Meyer B, Kim SL, David WP, Hüppe L, et al. (2017) Circadian Clock Involvement in Zooplankton Diel Vertical Migration. Current Biology 27(14): 2194-2201.e3.

18. Gupta NJ, Dwivedi V, Singh BP, Bhardwaj SK (2017) Orientation in migrating animals: role of biological clocks. In: Vinod Kumar(Ed.), Biological Timekeeping: Clocks, Rhythms and Behaviour, Springer, India, pp. 643-658.

19. Williams BG, Naylor E (1967) Spontaneously induced rhythm of tidal periodicity in laboratory-reared Carcinus. J Exp Biol 47(2): 229-234.

20. Korringa P (1957) Lunar periodicity. Geol Soc Am Memoir 67(1): 917934.

21. Kaiser TS, Heckel DG (2012) Genetic architecture of local adaptation in 
lunar and diurnal emergence times of the marine midge Cluniomarinus (Chironomidae, Diptera). PLoS ONE 7(2): e32092.

22. StillmanJH, Barnwell FH (2004) Relationship of daily and circatidal activity rhythms of the fiddler crab, Uca princeps, to the harmonic structure of semidiurnal and mixed tides. Marine Biol. 144(3): 473-482.

23. Zeng C, Abelloe P, Naylor E (1999) Endogenous tidal and semilunar moulting rhythms in early juvenile shore crabs Carcinusmaenas: implications for adaptation to a high intertidal habitat. Marine Ecology Progress Series 191: 257-266.

24. Alifierakis NS, Berry J (1980) Rhythmic egg-release in Littorinalittorea (Mollusca: Gastropoda). Journal of Zoology 190(3): 297-307.

25. Meisel DV, Byrne RA, Kuba M, Griebel U, Mather JA (2003) Circadian rhythms in Octopus vulgaris. Berliner Paläobiol. Abh 03: 171-177.

26. Newcomb JM, Kirouac EL, Naimie AA, Bixby KA, LEE C, et al. (2014)
Circadian Rhythms of Crawling and Swimming in the Nudibranch Mollusc Melibe leonine. Biol Bull 227(3): 263-273.

27. Caballes CF, Pratchett MS (2017) Environmental and biological cues for spawning in the crown-of-thorns starfish. Plos One 12(3): e0173964.

28. Peres R, Amaral FG, Marques AC, Neto JC (2014) Melatonin production in the sea star Echinaster brasiliensis (Echinodermata). Biol Bull 226(2): 146-151.

29. Ullrich-Lüter EM, Dupont S, Arboleda E, Hausen H, Arnonec MA, et al. (2011) Unique system of photoreceptors in sea urchin tube feet. Proceeding of the National Academy Sciences of the United States of America 108(20): 8367-8372.

30. Dong G, Dong S, Wang F, Tian X (2010) Effects of light intensity on daily activity rhythm of juvenile sea cucumber, Apostichopus japonicus (Selenka). Aquaculture Research 41(11): 1640-1647. 